\title{
The Superconducting Proximity Effect as a Tool to Investigate Metal Films and Interfaces.
}

\author{
D. Garrett, M. Zhang and G. Bergmann. \\ University of Southern California
}

November 3, 2018

\begin{abstract}
The superconducting proximity effect is measured in sandwiches of thin $\mathrm{Pb}$ films and the alkali metals $\mathrm{Cs}, \mathrm{Rb}, \mathrm{K}$ and $\mathrm{Na}$. The $T_{c^{-}}$ dependence provides information about the interface barriers between $\mathrm{Pb}$ and the alkalis. Such a barrier is particularly large in $\mathrm{Pb} / \mathrm{Cs}$ sandwiches. It is not due to impurities or oxydation. In the presence of a sufficiently strong barrier a special form of the Cooper limit can be applied to calculate the transition temperature of the sandwich.

PACS: 74.50.+r, 73.20.-r, 71.20.Dg
\end{abstract}




\section{Introduction}

When a thin superconducting film is covered with a normal conductor its transition temperature is lowered. This phenomenum is known as the "Superconducting Proximity Effect" (SPE), and it was in tensively studied in the 1960s and 70s [1], [2], [3], [4], [5], [6], [7] (see for example [8], ). (In those days one had the hope that an extrapolation of $T_{c}$ would yield a finite transition temperature for normal conductors such as the noble metals.) However, there has been a continuous interest in this effect over the years [9], [10], [11], [12], [13] which extended recently into superconductor-ferromagnetic metal sandwiches (see for example [14]). In the present paper we want to revive the SPE as a tool to investigate interfaces between metal films.

In recent years we have investigated the properties of thin alkali metal films [15], [16], [17],[18], [19], [20], [21], [22], [23]. We observed a number of properties which were quite unexpected:

- The Hall effect and resistance of thin Cs increased dramatically when the film was covered in situ with small concentrations of $(\mathrm{s}, \mathrm{p})$ impurities.

- Sandwiches of quench-condensed Fe covered with a film of Cs or K showed a mean free path in the alkali film which can be up to five times the thickness of the alkali film. This means that the electrons in the $\mathrm{Cs}$ or $\mathrm{K}$ are almost perfectly specularly reflected at the free surface and the interface with the Fe. The latter is quite surprizing since the Fe is very disordered and the electrons should be diffusively scattered at the interface.

- Recently we used the $\mathrm{SPE}$ in a $\mathrm{Pb} / \mathrm{K} / \mathrm{Pb}$ sandwich to study the local electronic properties of the $\mathrm{K}$ while it was coveraged with sub-monolayers $\mathrm{Pb}$. On the one hand the coverage of the $\mathrm{K}$ with $\mathrm{Pb}$ appeared to localize the electrons in the $\mathrm{K}$ while on the other hand the $T_{c}$-reduction of the first $\mathrm{Pb}$ film by the $\mathrm{K}$ remained unaltered.

The properties of interfaces and the ability of electrons to cross them are important in many physical phenomena and applications. To name one example for the latter, the giant magneto-resistance in magnetic multi-layers 
depends critically on the ability of the conduction electrons to cross "freely" from one film to the next. The goal of this paper is to investigate the SPE in $\mathrm{Pb} / \mathrm{Ak}$ films (in the following we use the symbol "Ak" as synonym for any of the four alkali metals $\mathrm{Cs}, \mathrm{Rb}, \mathrm{K}$ and $\mathrm{Na}$ ) and to show that the SPE is a suitable tool to obtain information about interfaces.

\section{Experiment}

In the present investigation we prepare sandwiches of $\mathrm{Pb} / \mathrm{Ak}$ at liquid helium temperatures. In most experiments we quench condense a $\mathrm{Pb}$ film of 13 to $14 \mathrm{~nm}$ thickness and a resistance of about $100 \Omega$. The $\mathrm{Pb}$ is then covered in several steps with an alkali metal. After each evaporation the film or sandwich is annealed, the original $\mathrm{Pb}$ film up to $40 \mathrm{~K}$ and the sandwiches up to $35 K$. Then the superconducting transition curve is recorded and the magneto-resistance and Hall resistance are measured at $9.5 K$ in the field range between $-7 T$ and $+7 T$. In these experiments one has to be careful that the edges of the $\mathrm{Pb}$ film are covered by the normal metal. Otherwise one obtains double transition curves. Fig.1 shows a set of transition curves for $\mathrm{Pb} / \mathrm{K}$ sandwiches.

In Fig.2a-d the dependence of $T_{c}$ on the coverage with $\mathrm{Cs}, \mathrm{Rb}, \mathrm{K}$ and $\mathrm{Na}$ is plotted. The full circles represent the experimentally measured $T_{c}$. The curves are discussed below.

\section{Theory and Discussion}

Werthamer [3] derived an implicit set of equations for the transition temperature of a sandwich of two superconductors. The superconductor with the lower transition temperature is generally called the normal conductor because at the $T_{c}$ of the sandwich it would be in the normal conducting state. In this theory the gap function $\Delta(\mathbf{r})$ in the superconductor is proportional to $\cos \left[k_{s}\left(z+d_{s}\right)\right]$ and in the normal conductor proportional to $\cosh \left[k_{n}\left(z-d_{n}\right)\right]$, with $z=0$ at the interface. The $k_{s, n}$ are the inverse superconducting coherence lengths. For disordered (dirty) metal films they are 
given by

$$
\begin{aligned}
\ln \left(\frac{T_{s}}{T_{c}}\right) & =\chi\left(\xi_{s}^{2} k_{s}^{2}\right), \ln \left(\frac{T_{c}}{T_{n}}\right)=-\chi\left(-\xi_{n}^{2} k_{n}^{2}\right) \\
\chi(z) & =\Psi\left(\frac{1}{2}+\frac{1}{2} z\right)-\Psi\left(\frac{1}{2}\right) \\
\xi^{2} & =\frac{\hbar v_{F} l}{6 \pi k_{B} T_{c}}=\frac{\pi \hbar k_{B}}{6 e^{2} T_{c}} \frac{\sigma}{\gamma}=D \tau_{T} \\
\tau_{T} & =\frac{\hbar}{2 \pi k_{B} T_{c}}
\end{aligned}
$$

Here $T_{s}, T_{n}$ and $T_{c}$ are the transition tempertures of the two superconductors $\left(T_{s}>T_{n}\right)$ and the sandwich $\xi_{s, n}$ are the thermal coherence lengths, $\sigma$ the conductivity, $\gamma$ the Sommerfeld constant (which stands for the density of states), $D$ the diffusion constant, $v_{F}$ the Fermi velocity, $\tau_{T}$ the thermal time, and $\psi(x)$ the digamma function.

At the interface Werthamer originally used the boundary condition that $\frac{1}{\Delta} \frac{d \Delta}{d z}$ is continous. de Gennes [24] derived instead that $\Delta /(N V)$ and $(D / V)(d \Delta / d z)$ are continous at the interface, the latter only in the "dirty" limit. The combined function $(D / V)(d \Delta / d z) / \Delta /(N V)=D N(d \Delta / d z) / \Delta$ is then continous as well. This yields boundary condition

$$
N_{s} \xi_{s}^{2} k_{s} \tan \left(k_{s} d_{s}\right)=N_{n} \xi_{n}^{2} k_{n} \tanh \left(k_{n} d_{n}\right)
$$

which determine $T_{c}$. The equations (1) and (2) together are often called the Wertheimer-deGennes theory. We will abriviate the theory as the WGtheory.

The WG-theory is restricted to the dirty case, where the mean free path of the conduction electrons is much smaller than the superconducting coherence length. It is interesting to note that Werthamer's equations require only three parameter of each superconductor besides the thickness: the transition temperture, the resistivity and the density of states (Sommerfeld constant).

Deutscher and DeGennes [8] point out the calculation for $\Delta(x)$ within each superconductor uses only one root for the decay constant (the smallest Matsubara frequency). Nevertheless Werthamer's equations were successfully applied to a number of experimental results (see for example [8], [6]). Therefore it is interesting to compare the experimental results with the WGtheory. 
In Fig.2a-d we calculate the transition temperature with the WG-theory for the different $\mathrm{Pb} / \mathrm{Ak}$ sandwiches. As far as we know the alkali metals are not superconducting. On the other hand the WG-theory requires a finite transition temperature $T_{n}$ for both metal films. Therefore we treat the alkali metals as hypothetical superconductors with a transition tempereture of $T_{n}=10^{-5} \mathrm{~K}$. (It turns out that the choice of $T_{n}=10^{-2} \mathrm{~K}$ does not make any difference in the theoretical transition temperatures at the experimental thicknesses of the alkali metals. Only in an extremely small thickness range of the alkali metals $d_{n} \rightarrow 0$ does one find a small difference (which, however, influences the initial slope $d T_{c} / d d_{n}$ at zero thickness of the alkali metal.) We recognize from Fig.2 that the heavy alkali metals such as Cs show a large deviation from the WG-theory while the sandwich with the light $\mathrm{Na}$ is relatively close to the theoretical curve. For $\mathrm{Pb} / \mathrm{Rb}$ and $\mathrm{Pb} / \mathrm{K}$ the region of small alkali thickness appears to be reasonably reproduced by the WT.

The initial slope $d T_{c} / d d_{n}$ of $T_{c}$ versus the thickness of the normal metal at $d_{n}=0$ can be derived from Werthamer's theory and is given by

$$
\frac{d_{s}}{T_{s}} \frac{d T_{c}}{d d_{n}}=-\frac{\pi^{2}}{4} \frac{N_{n}}{N_{s}} \chi^{-1}\left(-\ln \left(\frac{T_{s}}{T_{n}}\right)\right)
$$

The expression $\chi^{-1}\left(-\ln \left(\frac{T_{s}}{T_{n}}\right)\right)$ approaches the value one for a large $\left(T_{s} / T_{n}\right)$ ratio, but for $T_{n}=10^{-2} K\left(\right.$ with $\left.T_{s}=7.2 K\right)$ one has $\chi^{-1}\left(-\ln \left(7.2 / 10^{-2}\right)\right)=$ -0.75 . Since the Werthamer value of the initial slope depends strongly on the choice of $T_{n}$ while at finite thickness there is no such dependence we prefer to compare the value $\Delta T_{c} / \Delta d_{n}$ between experiment and theory. Here $\Delta d_{n}$ is the smallest evaporated film thickness of the alkali metal and $\Delta T_{c}$ is the $T_{c^{-}}$ reduction due to this alkali coverage. (We will still call the ratio $\Delta T_{c} / \Delta d_{n}$ the initial slope.) These values are collected in Table I for the four alkali sandwiches studied. For the alkali metals we used the density of states as given in Ashcroft and Merman [25]. In collumn three the factor $N^{*} / N_{0}$, the ratio between the (experimental) density of states $N^{*}$ and the free electron value $N_{0}$, is given. For the very disordered $\mathrm{Pb}$ we used a density ratio of 2.5 because disordered $\mathrm{Pb}$ has an even higher gap ratio $2 \Delta_{0} / k_{B} T_{c}=4.6$ than that of pure $\mathrm{Pb}$ and therefore a larger value of $(1+\lambda)$ than pure (annealed) $\mathrm{Pb}$, where $(1+\lambda)$ is the electron-phonon enhancement factor. 


\begin{tabular}{|l|l|l|l|l|l|l|}
\hline $\begin{array}{l}\text { Alkali } \\
\text { metal }\end{array}$ & $\begin{array}{l}\text { exper. } \\
\text { code }\end{array}$ & $\frac{N^{*}}{N_{0}}$ & $\mathrm{~d}_{\mathrm{n}}(n m)$ & $\left(\frac{\Delta T_{c}}{\Delta d_{n}}\right)_{\exp }$ & $\left(\frac{\Delta T_{c}}{\Delta d_{n}}\right)_{\mathrm{Wh}}$ & $\frac{\tau_{T_{c}}}{\tau_{f}}$ \\
\hline $\mathrm{Na}$ & $\mathrm{GI}$ & 1.3 & 2.18 & 0.280 & 0.32 & - \\
\hline $\mathrm{K}$ & $\mathrm{GF}$ & 1.2 & 2.04 & 0.235 & 0.27 & 0.08 \\
\hline $\mathrm{Rb}$ & $\mathrm{GC}$ & 1.3 & 1.77 & 0.249 & 0.25 & 0.052 \\
\hline $\mathrm{Cs}$ & $\mathrm{OC}$ & 1.5 & 2.29 & 0.175 & 0.31 & .035 \\
\hline & & & & & & \\
\hline
\end{tabular}

Table I : The experimental value of $\frac{\Delta T_{c}}{\Delta d_{n}}$ and the prediction according to Werthamer's thoery for the different alkali sandwiches.

The initial slope does not depend on the mean free path in either metal. The same is true for the small values of $\Delta d_{n}$. In Werthamer's theory it only depends on the ratio of the density of states. For the $\mathrm{Pb}$ sandwiched with $\mathrm{Na}, \mathrm{K}$ and $\mathrm{Rb}$ the experimental initial slope agrees within $15 \%$ with the prediction of the WG-theory. For Cs the experimental initial slope is much smaller than Werthamer's prediction. We believe that this is caused by some kind of barrier at the $\mathrm{Pb}-\mathrm{Cs}$ interface.

From the experimental results the deviation between experiment and WG-theory for the $\mathrm{Pb} / \mathrm{Cs}$ sandwich is particularly striking. One might object that the small reduction in $T_{c}$ is caused by an oxide layer between the $\mathrm{Pb}$ and the Cs. We exclude this interpretation for several reasons: (i) The results have been reproduced in several experiments, (ii) the other alkali metal such as $\mathrm{Na}$ and $\mathrm{K}$ would show an even stronger tendency to oxidation.

To investigate this question we prepare a sandwich in which the $\mathrm{Pb}$ is first covered with $2 \mathrm{~nm}$ of $\mathrm{Na}$ and afterwards with Cs of increasing thickness. In Fig.3 the transition temperature of the $\mathrm{Pb} / \mathrm{Na} / \mathrm{Cs}$ sandwich is plotted versus the alkali thickness $d_{N a}+d_{C s}$. For comparison the $T_{c}$-dependence of the $\mathrm{Pb} / \mathrm{Cs}$ and the $\mathrm{Pb} / \mathrm{Na}$ sandwiches are shown in the same figure. One realizes that the transition temperature of the $\mathrm{Pb} / \mathrm{Na} / \mathrm{Cs}$ sandwich is much closer to the $\mathrm{Pb} / \mathrm{Na}$ sandwich than to the $\mathrm{Pb} / \mathrm{Cs}$ sandwich. This demonstrates that, after bridging the contact between the $\mathrm{Pb}$ and the $\mathrm{Cs}$, one finds a similar $T_{c}$-reduction as in the $\mathrm{Pb} / \mathrm{Na}$ sandwich. It is not the electronic properties of the Cs which yield the small initial slope and the weak reduction of $T_{c}$ in the $\mathrm{Pb} / \mathrm{Cs}$ sandwich. Instead the interface between $\mathrm{Pb}$ and $\mathrm{Cs}$ must create some kind of obstacle which makes it harder for the electrons to cross the 
interface. About the nature of this obstacles one can only speculate at the present time. However, we are quite sure that it is not due to dirt or oxygen. And neither the $\mathrm{Pb}$ nor the $\mathrm{Cs}$ are individually responsible for the obstacle because in the $\mathrm{Pb} / \mathrm{Na} / \mathrm{Cs}$ sandwich the more agressive $\mathrm{Na}$ faces each of the two and the obstacle (at each interface) is either absent or much smaller than in the $\mathrm{Pb} / \mathrm{Cs}$ interface.

If one compares the experimental $T_{s}$ curves for $\mathrm{Pb}$ with $\mathrm{Cs}, \mathrm{Rb}, \mathrm{K}$ and $\mathrm{Na}$ with the theory of Wertheimer one recognizes right away that the deviation between experiment and the Wertheimer theory decreases in going from Cs to Na. It is not obvious that the Wertheimer theory has to be correct for our $\mathrm{Pb} / \mathrm{Ak}$ sandwiches; in fact this theory may be not appropriate at all for the alkali metals. But the $\mathrm{Pb} / \mathrm{Na} / \mathrm{Cs}$ sandwich demonstrates for the $\mathrm{Pb} / \mathrm{Cs}$ case that the deviation is not due to the Cs but the interface. Therefore it is suggestive that all the interfaces $\mathrm{Pb} / \mathrm{Ak}$ present some kind of obstacles for the transmission of the electrons through the interface, where the strength of the obstacle decreases in going from $\mathrm{Pb} / \mathrm{Cs}$ to $\mathrm{Pb} / \mathrm{Na}$. This does not contradict the fact the experimental initial slopes for the three alkali metals agree quite well with the Wertheimer theory. This we demonstrate for the Cooper limit of the SPE.

The Cooper limit applies when the gap function $\Delta(\mathbf{r})$ can be treated as constant in each metal of the film. This is fulfilled when the thickness of each metal is much smaller than its coherence length. If the exchange of electrons between the superconductor and the normal conductor is strongly reduced then the Cooper limit is valid in a considerably larger thickness range. As discussed in the Appendix A one can formulate the following condition: Mark all electrons in a small energy range about the Fermi surface in the superconductors. Follow their density distribution as a function of time while they can propagate into the normal conductor. If their density is at all times reasonably constant within the superconductor, then the gap function $\Delta(\mathbf{r})$ will be a constant $\Delta_{s}$ (at the transition temperature) and the Cooper case applies. This can, for example, happen for relatively thick films if the escape time from the superconductor into the normal conductor is long.

In Appendix A we derive the Cooper limit with a barrier between the two metals from a special version of the linear gap equation. The theory requires one fitting parameter, the transmission rate $1 / \tau_{s n}$ from the $\mathrm{Pb}$ into the alkali film. The resulting theoretical curves are plotted in Fig.2a,b,c as full curves and the fitted rates $\tau_{T_{c}} / \tau_{t}$ are collected in table I. They describe 
the behavior of $T_{c}$ at finite thickness $d_{n}$ of the alkali metal films quite well, in particular the saturation in the $\mathrm{Pb} / \mathrm{Cs}$ sandwich. Surprisingly the WGtheory gives a better fit for the sandwiches with $\mathrm{Rb}, \mathrm{K}$ and $\mathrm{Na}$ at very small alkali thicknesses.

For low transmission rate $1 / \tau_{s n}$ through the interface and large normal conductor thickness the electrons of the superconductor escape with a rate of $1 / \tau_{s n}$ from the superconductor. This acts as a pair-weakening rate of $1 / \tau_{s n}$ and reduces the transition temperature as

$$
\frac{\Delta T_{c}}{T_{s}}=\frac{\pi^{2}}{2} \frac{\hbar}{2 \pi k_{B} T_{c}} \frac{1}{\tau_{n s}}
$$

Ashida et al. [26] calculated the transition of superconductor - normal conductor sandwiches with barries inbetween. They described the strength of the barrier by the coefficient of reflectivity $R$ at the interface. (In the absence of a barrier the value of $R$ is not zero but given by density of states ratio). They find in the limit $(R-1)<<1$ for the transition temperature

$$
\frac{\Delta T_{c}}{T_{s}}=\frac{\pi^{2}}{16} \frac{\hbar}{2 \pi k_{B} T_{c}} \frac{(1-R) v_{s}}{d_{s}}
$$

The two result are compared in the appendix.

A full numerical solution of the linear gap equation (6) with an adjustable transmission rate between the $\mathrm{Pb}$ and the alkali film would be desirable. Presently we are developing the software for such a solution.

Acknowledgment: The research was supported by NSF Grant No. DMR0124422 . 


\section{Appendix}

In close vicinity of the transition temperature the superconducting gap function $\Delta(\mathbf{r})$ is very small and the "gap equation" can be linearized [27].

$$
\begin{aligned}
\Delta(\mathbf{r}) & =V(\mathbf{r}) \int d^{3} \mathbf{r}^{\prime} \frac{1}{\tau_{T}} \sum_{\omega} H_{\omega}\left(\mathbf{r}, \mathbf{r}^{\prime}\right) \Delta\left(\mathbf{r}^{\prime}\right) \\
H_{\omega}\left(\mathbf{r}, \mathbf{r}^{\prime}\right) & =G_{\omega}^{*}\left(\mathbf{r}, \mathbf{r}^{\prime}\right) G_{\omega}\left(\mathbf{r}, \mathbf{r}^{\prime}\right) \\
\frac{1}{\tau_{T}} & =\frac{2 \pi k_{B} T}{\hbar}
\end{aligned}
$$

Here $\Delta(\mathbf{r})$ is the gap function at the position $\mathbf{r}, \omega_{n}=(n+1 / 2) / \tau_{T}$ are the Matsubara frequencies, $N\left(\mathbf{r}^{\prime}\right)$ is the (BCS)-density of states for one spin direction, $V(\mathbf{r})$ is the effective electron-electron interaction at position $\mathbf{r}$. The function $H_{\omega}\left(\mathbf{r}, \mathbf{r}^{\prime}\right)$ is the product of the two single electron Green functions $G_{\omega}\left(\mathbf{r}, \mathbf{r}^{\prime}\right)$ of a Cooperon. Following de Gennes [24], [8], Lueders [28] and one of the authors [29] we use a different approach to solve the gap equation. Since the $G_{w}$ represent the amplitude of an electron traveling (at finite temperarture) from $\mathbf{r}^{\prime}$ to $\mathbf{r}$ the product $G_{\omega}^{*}\left(\mathbf{r}, \mathbf{r}^{\prime}\right) G_{\omega}\left(\mathbf{r}, \mathbf{r}^{\prime}\right)$ describes the pair amplitude of the Cooperon to travel from $\mathbf{r}^{\prime}$ to $\mathbf{r}$. Since the two $G_{w}$ are conjugate complex to each other and are independent of the spin direction, the pair amplitude is identical to the probability of a single electron to travel from $\mathbf{r}^{\prime}$ to $\mathbf{r}$. The function $H_{\omega}\left(\mathbf{r}, \mathbf{r}^{\prime}\right)$ can be expressed by the function $F\left(\mathbf{r}, 0 ; \mathbf{r}^{\prime}, t^{\prime}\right)$ which gives the probability of an electron to travel from $\mathbf{r}^{\prime}$ to $\mathbf{r}$ during the time interval $\left|t^{\prime}\right|$ (departing at $\mathbf{r}^{\prime}$ at the negative time $t^{\prime}$ and arriving ar $\mathbf{r}$ at the time $t=0)$ while it experiences an exponential damping of $\exp \left(2|\omega| t^{\prime}\right)$.

$$
H_{\omega}\left(\mathbf{r}, \mathbf{r}^{\prime}\right)=\int_{-\infty}^{0} d t^{\prime} e^{2|\omega| t^{\prime}} F\left(\mathbf{r}, 0 ; \mathbf{r}^{\prime}, t^{\prime}\right) N\left(\mathbf{r}^{\prime}\right)
$$

This yields the gap equation

$$
\Delta(\mathbf{r})=V(\mathbf{r}) \int d^{3} \mathbf{r}^{\prime} \int_{-\infty}^{0} \frac{d t^{\prime}}{\tau_{T}} \sum_{\omega} e^{2|\omega| t^{\prime}} F\left(\mathbf{r}, 0 ; \mathbf{r}^{\prime}, t^{\prime}\right) N\left(\mathbf{r}^{\prime}\right) \Delta\left(\mathbf{r}^{\prime}\right)
$$

The function $\sum_{\omega} e^{-2|\omega| t}$ describes the exponentially decaying coherence of the Cooperons. 
This equation has a very transparent interpretation. From a given position $\mathbf{r}^{\prime}$ and at a given time $t^{\prime}<0$ there are $N\left(\mathbf{r}^{\prime}\right) \Delta\left(\mathbf{r}^{\prime}\right)$ electrons (representing the pair amplitude) propagating into all directions of the metal and experiencing scattering. Along the way their number decays exponentially as $\sum_{\omega} e^{-2|\omega| t}$. At a given time, for example at $t=0$ and at each position $\mathbf{r}$ one sums the contribution of all surviving electrons from all $\left(\mathbf{r}^{\prime}, t^{\prime}\right)$, forming the integral $\int d \mathbf{r}^{\prime} \int_{-\infty}^{0} d t^{\prime}$ and multiplies the result with $V(\mathbf{r})$. The result has to reproduce self-consistantly everywhere the gap function $\Delta(\mathbf{r})$.

If one is dealing with a time dependent gap function the derivation of a time-dependent Ginzburg-Landau equation from (6) is straight forward by replacing the time 0 by $t$ and $\Delta(\mathbf{r}), \Delta\left(\mathbf{r}^{\prime}\right)$ by $\Delta(\mathbf{r}, t), \Delta\left(\mathbf{r}^{\prime}, t^{\prime}\right)[29]$.

Since we are here not interested in time-dependent gap functions we may shift the time scale by starting the propagation at $\mathbf{r}^{\prime}$ at the time $t=0$ and arriving at $\mathbf{r}$ at the time $t>0$. Then we have

$$
\Delta(\mathbf{r})=V(\mathbf{r}) \int d^{3} \mathbf{r}^{\prime} \int_{0}^{\infty} \frac{d t^{\prime}}{\tau_{T}} \sum_{\omega} e^{-2|\omega| t} F\left(\mathbf{r}, t ; \mathbf{r}^{\prime}, 0\right) N\left(\mathbf{r}^{\prime}\right) \Delta\left(\mathbf{r}^{\prime}\right)
$$

The great advantage of this description is the fact that a major part of solving the gap equation involves only the dynamics of the conduction electrons. We demonstrate this first for the Cooper limit of an SN-sandwich.

Cooper case without barrier: We assume that the electron-electron interaction in the normal metal is zero $V_{n}=0$. In this case the gap function is only non-zero in the superconductor and both $\mathbf{r}^{\prime}$ and $\mathbf{r}$ lie in the superconducting film. Since in the Cooper limit $\Delta\left(\mathbf{r}^{\prime}\right)$ is constant and has the value $\Delta_{s}$ in the superconducting film one obtains for the gap equation

$$
\Delta_{s}=V_{s} \int_{S} d^{3} \mathbf{r}^{\prime} \int_{0}^{\infty} \frac{d t}{\tau_{T}} \sum_{\omega} e^{-2|\omega| t} F\left(\mathbf{r}, t ; \mathbf{r}^{\prime}, 0\right) N_{s} \Delta_{s}
$$

The integration $d \mathbf{r}^{\prime}$ extends only over the superconducting film. The electron propagation function $F\left(\mathbf{r}, t ; \mathbf{r}^{\prime}, 0\right)$ describes the probability of $\left(N\left(\mathbf{r}^{\prime}\right) \Delta_{s}\right.$ electrons which start at $\left(\mathbf{r}^{\prime}, 0\right)$ (in the superconductor) and arrive at $t$ at the position $\mathbf{r}$ (in $\mathrm{S}$ ). (Although the start and end points of the electron path lie in the superconductor the path can extend into the normal conductor as well.) Since the electrons propagate roughly with the Fermi velocity perpendicular to the film plane the time to move the distance $d_{s}$ or $d_{n}$ is very short compared with the thermal coherence time $\tau_{T}=\hbar /\left(2 \pi k_{B} T\right)$. Therefore any 
electron - independently of where it started - will be found, after a very short time, in the superconductor with the probability $p_{s}=\frac{d_{s} N_{s}}{d_{s} N_{s}+d_{n} N_{n}}$. Therefore one obtains $\int_{S} d^{3} \mathbf{r}^{\prime} F\left(\mathbf{r}, t ; \mathbf{r}^{\prime}, 0\right)=\frac{d_{s} N_{s}}{d_{s} N_{s}+d_{n} N_{n}}$ for (almost) any time $t$. This yields the equation

$$
\begin{aligned}
\Delta_{s} & =V_{s} N_{s} \frac{d_{s} N_{s}}{d_{s} N_{s}+d_{n} N_{n}} \int_{0}^{\infty} \frac{d t^{\prime}}{\tau_{T}} \sum_{\omega} e^{2|\omega| t^{\prime}} \Delta_{s} \\
1 & =V_{s} N_{s} \frac{d_{s} N_{s}}{d_{s} N_{s}+d_{n} N_{n}} \frac{1}{\tau_{T}} \sum \frac{1}{2|\omega|} \\
\text { with } \frac{1}{\tau_{T}} \sum \frac{1}{2|\omega|} & =\sum_{n=0}^{n_{c}} \frac{1}{n+\frac{1}{2}}
\end{aligned}
$$

The equation (8) is the well known Cooper condition for the transition temperature of the sandwich where the attractive interaction is given by $V_{\text {eff }}=$ $V_{s} \frac{d_{s} N_{s}}{d_{s} N_{s}+d_{n} N_{n}}$, resulting in the transition temperature $T_{c}=1.14 \Theta_{D} \exp \left[-1 / N_{s} V_{\text {eff }}\right]$. For the initial slope in the Cooper limit one obtains then

$$
\frac{d_{s}}{T_{s}} \frac{d T_{c}}{d d_{n}}=-\frac{1}{V_{s} N_{s}} \frac{N_{n}}{N_{s}}
$$

It should be pointed out that the initial slope in the Cooper limit does not exactly agree with the result by Werthamer, emphasizing that Werthamer's solution contains approximations.

Cooper case with barrier: In the next step we consider the Cooper case with a barrier between the superconducting and the normal metal film. An electron in $\mathrm{S}$ has a finite transmission rate through the interface which is proportional to the density of states in the normal conductor $N_{n}$ and inversely proportional to the thickness of the superconductor $d_{s}$.

$$
\frac{1}{\tau_{s n}}=\alpha \frac{1}{d_{s}} N_{n}
$$

We follow the fate of such an electron, denoting the probability to be in $\mathrm{S}$ or $\mathrm{N}$ as $n_{s}$ and $n_{n}$, where $\left(n_{s}+n_{n}\right)=1$. Then we have

$$
\begin{aligned}
\frac{d n_{s}}{d t} & =-\alpha n_{s} \frac{1}{d_{s}} N_{n}+\alpha n_{n} \frac{1}{d_{n}} N_{s} \\
\frac{d n_{n}}{d t} & =\alpha n_{s} \frac{1}{d_{s}} N_{n}-\alpha n_{n} \frac{1}{d_{n}} N_{s}
\end{aligned}
$$


The solution of this equation is

$$
\begin{aligned}
n_{s} & =n_{\infty}+n_{\Delta} \exp \left(-\frac{t}{\tau_{r}}\right) \\
\text { with } n_{\infty} & =\frac{N_{s} d_{s}}{\left(N_{n} d_{n}+N_{s} d_{s}\right)}, n_{\Delta}=\frac{d_{n} N_{n}}{\left(N_{n} d_{n}+N_{s} d_{s}\right)} \\
\frac{1}{\tau_{r}} & =\alpha\left(\frac{N_{n}}{d_{s}}+\frac{N_{s}}{d_{n}}\right)=\frac{1}{\tau_{s n}}+\frac{1}{\tau_{n s}}
\end{aligned}
$$

where $\tau_{r}^{-1}$ is the Cooperon relaxation rate. It the sum of the transmission rates through the interface in both directions. This yields the following gap equation

$$
\Delta_{s}=V_{s} N_{s} \int_{0}^{\infty} \frac{d t}{\tau_{T}} \sum_{\omega}\left(n_{\infty} e^{-2|\omega| t}+n_{\Delta} e^{-\left(2|\omega|+\frac{1}{\tau_{r}}\right) t}\right) \Delta_{s}
$$

or

$$
1=\frac{V_{s} N_{s}}{d_{s} N_{s}+d_{n} N_{n}}\left(d_{s} N_{s} \sum_{n=0}^{n_{c}} \frac{1}{\left(n+\frac{1}{2}\right)}+d_{n} N_{n} \sum_{n=0}^{n_{c}} \frac{1}{\left(n+\frac{1}{2}+\frac{1}{2} \frac{\tau_{T}}{\tau_{r}}\right)}\right)
$$

This equation shows already qualitatively the effect of the barrier for the two limiting cases:

- weak transmission, $\tau_{T} / \tau_{r}<1$ : In this case the sum in the second term on the right side of equation (10) is almost the sum as the first sum. One obtains almost the transition temperature of the pure superconductor. $T_{c}$ is given by the inplicit equation

$$
1=V_{s} N_{s} \sum_{n=0}^{n_{c}} \frac{1}{n+\frac{1}{2}}-V_{s} N_{s} \frac{d_{n} N_{n}}{\left(N_{n} d_{n}+N_{s} d_{s}\right)}\left[\psi\left(\frac{1}{2}+\frac{1}{2} \frac{\tau_{T}}{\tau_{r}}\right)-\psi\left(\frac{1}{2}\right)\right]
$$

- strong transmission, $\tau_{T} / \tau_{r}>1$ : In this case one obtains

$$
1=V_{s} N_{s} \frac{d_{s} N_{s}}{\left(N_{n} d_{n}+N_{s} d_{s}\right)} \sum_{n=0}^{n_{c}} \frac{1}{n+\frac{1}{2}}+\frac{d_{n} N_{n}}{\left(N_{n} d_{n}+N_{s} d_{s}\right)} \sum_{n=0}^{n_{c}} \frac{1}{n+\frac{1}{2}+\frac{1}{2} \frac{\tau_{T}}{\tau_{r}}}
$$

where the second term on the right side can be neglected for large $\tau_{T} / \tau_{r}$ and the remaining part yields just the Cooper transition temperature without a barrier. 
We perform the numeric solution of equation (10) for the $\mathrm{Pb} / \mathrm{Cs}$ sandwich. For this purpose one has to solve the equation

$$
d_{s} N_{s}\left(\sum_{n=0}^{n_{c}} \frac{1}{\left(n+\frac{1}{2}\right)}-\frac{1}{V_{s} N_{s}}\right)+d_{n} N_{n}\left(\sum_{n=0}^{n_{c}} \frac{1}{\left(n+\frac{1}{2}+\frac{1}{2} \frac{\tau_{T}}{\tau_{r}}\right)}-\frac{1}{V_{s} N_{s}}\right)=0
$$

with

$$
\begin{aligned}
n_{c} & =\frac{\Theta_{D}}{2 \pi T_{c}}, n_{c 0}=\frac{\Theta_{D}}{2 \pi T_{s}} \\
\rho & =\frac{\tau_{T}}{\tau_{r}}=\frac{\tau_{T}}{\tau_{s n}}\left(1+\frac{d_{s} N_{s}}{d_{n} N_{n}}\right) \\
\frac{1}{V_{s} N_{s}} & =\sum_{n=0}^{n_{c 0}} \frac{1}{\left(n+\frac{1}{2}\right)}
\end{aligned}
$$

The resulting $T_{c}$-values are plotted in Fig.2a and Fig.3 as full curves. For the transmission rate we used the value $1 / \tau_{s n}=.035 / \tau_{T_{s}}=.035 * 2 \pi k_{B} T_{s} / \hbar$ $=2.0 \times 10^{11} s^{-1}$ or $\tau_{s n}=5 \times 10^{-12} s$. $\left(1 / \tau_{s n}\right.$ is transmission rate for an electron in the $\mathrm{Pb}$ film to escape into the $\mathrm{Cs}$ film). It is much longer than the superconducting time constant $\tau_{T_{s}}=\hbar /\left(2 \pi k_{B} T_{s}\right)=1.7 \times 10^{-13} s$ and the ballistic flight time of the $\mathrm{Pb}$ electrons with (normalized) Fermi velocity (in z-direction): $\tau_{b}=2 d / v_{F}^{*}=4 \times 10^{-14} s$.

Similar evaluations are performed for the $\mathrm{Pb} / \mathrm{Rb}$ and $\mathrm{Pb} / \mathrm{K}$ sandwiches. The transmission rate ratios $\tau_{T_{s}} / \tau_{s n}$ are included in table I. For the $\mathrm{Pb} / \mathrm{Na}$ sandwich the agreement was not sufficient to include it in the Fig.2d. All the theoretical curves for the Cooper case with barrier yield a too large initial slope. For small thicknesses of the normal conductor the relaxation rate becomes arbitrarily large according to relation (9). This is unphysical, however, because the electron needs at least the ballistic flight time $\tau_{b}$ to cross over from the superconductor into the normal conductor. Therefore this range has to be excluded from the evaluation. Going from $\mathrm{Pb} / \mathrm{Cs}$ to $\mathrm{Pb} / \mathrm{Na}$ the alkali thickness range in which the barrier approach is not appropriate increases.

Ashida et al. [26] calculated the transition temperature of SN-sandwiches with barriers in-between.. They characterize the strength of the barrier by the coefficient of reflectivity $R$ at the interface. (In the absence of a barrier 
the value of $R$ is not zero but given by density of states ratio). They find in the limit $(R-1)<<1$ for the transition temperature (in our notation)

$$
\frac{\Delta T_{c}}{T_{s}}=\frac{\pi^{2}}{16} \frac{\hbar}{2 \pi k_{B} T_{c}} \frac{(1-R) v_{s}}{d_{s}}
$$

This limit $(R-1)<<1$ corresponds to in our notation to the limit $1 / \tau_{s n}<<1 / \tau_{T}$. If we follow Ashida et al. and expand 12 in terms of the pair breaking parameter $\rho$ we obtain

$$
\begin{gathered}
\sum_{n=0}^{n_{c}} \frac{1}{\left(n+\frac{1}{2}+\frac{1}{2} \rho\right)} \approx \sum_{n=0}^{n_{c}} \frac{1}{n+\frac{1}{2}}-\frac{\rho}{2} * \frac{1}{2} \pi^{2} \\
\left(\frac{d_{n} N_{n}}{d_{s} N_{s}}\right)^{-1}=-1+\frac{\frac{\rho}{2} * \frac{1}{2} \pi^{2}}{\left(\sum_{n=0}^{n_{c}} \frac{1}{\left(n+\frac{1}{2}\right)}-\frac{1}{V_{s} N_{s}}\right)}
\end{gathered}
$$

which yields

$$
\ln \left(\frac{T_{s}}{T_{n}}\right)=\frac{\pi^{2}}{4} \frac{\tau_{T}}{\tau_{s n}}
$$

or

$$
\frac{\Delta T_{c}}{T_{s}}=\frac{\pi^{2}}{4} \frac{\hbar}{2 \pi k_{B} T_{c}} \frac{1}{\tau_{s n}}
$$

The calculation of $\overline{1 / \tau_{s n}}$ in terms of $R$ is straight forward

$$
\frac{\overline{1}}{\tau_{s n}}=\frac{\int_{0}^{1} \frac{v_{F} \cos \theta}{2 d_{s}}(1-R) d(\cos \theta)}{\int_{0}^{1} d(\cos \theta)}=\frac{v_{F}(1-R)}{4 d_{s}}
$$

This expansion yields a perfect agreement between the results of Ashida et al. and ours. However, the result is somewhat surprising since the $T_{c}$ does not depend on the thickness of the normal conductor. Our numerical evaluation shows that this is not quite correct. The reason is the following:

The value of $\rho=\frac{\tau_{T}}{\tau_{r}}=\frac{\tau_{T}}{\tau_{s n}}\left(1+\frac{d_{s} N_{s}}{d_{n} N_{n}}\right)$ diverges for small thickness of the normal conductor, always reaching the limit of strong transmission for $d_{n} \rightarrow 0$. That means that at small thickness of the normal conductor one expects the full Cooper reduction of the transition temperature. This means in other words that even for $(R-1)<<1$ the expansion by Ashida et al. 
has to exclude the range of very small normal conductor thickness. When $d_{n}$ increases the transmission into the normal conductor becomes less effective, the superconducting film behaves more isolated and the $T_{c}$-reduction is much less effective. This is the experimental observation, in particular for the $\mathrm{Pb} / \mathrm{Cs}$ sandwich. 


\section{$5 \quad$ Figures}

Fig.1: The superconducting transition curves for $\mathrm{Pb}$ with different coverages of $\mathrm{K}$. 
Fig.2a-d: The transition temperatures of $\mathrm{Pb} / \mathrm{Ak}$ multilayers as a function of the alkali thickness. The dotted curves are calculated with the theory by Werthamer. For $\mathrm{Pb} / \mathrm{Cs}, \mathrm{Pb} / \mathrm{Rb}$ and $\mathrm{Pb} / \mathrm{K}$ the full curves are calculated within the Cooper model with a barrier between the $\mathrm{Pb}$ and the alkali film. For $\mathrm{Pb} / \mathrm{Na}$ the full curve is a guide to the eye. 
Fig.3: The transition temperatures of $\mathrm{Pb} / \mathrm{Na} / \mathrm{Cs}$ multilayers as a function of the alkali thickness (full circles). The transition temperatures of the $\mathrm{Pb} / \mathrm{Cs}$ sandwiches (up triangles) and the $\mathrm{Pb} / \mathrm{Na}$ sandwiches (down triangles) are shown for comparison. The full curve for the $\mathrm{PbCs}$ is calculated in the Cooper model with a barrier between the $\mathrm{Pb}$ and $\mathrm{Cs}$ films. 


\section{References}

[1] P.Hilsch and R.Hilsch, Naturwissenschaften 48, 549 (1961)

[2] P.Hilsch and R.Hilsch, Z.Physik 180, 10 (1964)

[3] N.R.Werthamer, Phys.Rev. 132, 2440 (1963)

[4] P.G.de Gennes and E.Guyon, Phys.Letters 3, 168 (1963)

[5] J.J.Hauser, H.C.Theurer and N.R.Werthamer, Phys.Rev. 136, A 637 (1964)

[6] G.Bergmann, Z.Physik, 187, 395 (1965)

[7] G.v.Minigerode, Z.Physik 192, 379 (1966)

[8] G.Deutscher and P.G.DeGennes, Superconductivity, ed. R.D.Parks Marcel Decker, Inc, New York, 1969, p. 1005

[9] A.Z.Zaitsev, Physica C. 185-189, 2539 (1991)

[10] A.Z.Zaitsev, Physica B. 203, 274 (1997)

[11] A.F.Volkov and A.V.Zaitsev, Phys.Rev. B 53, 9267 (1996)

[12] R.G.Mints and I.B.Shapiro, Phys.Rev. B57, 10318 (1998) 
[13] A.Volkovyz, N.Allsoppy and C J Lamberty, J.Phys.: Cond.Matter 8, 45 (1996)

[14] M.A.Sillanp, T.T.Heikkil, Europhys.Lett., 56(4), 590 (2001)

[15] H.Beckmann, T.Fulmer, D.Garrett, M.Hossain and G.Bergmann, Phys.Rev. B59, 7724 (1999)

[16] H.Beckmann and G.Bergmann, Eur.Phys.J B 13, 495 (2000)

[17] H.Beckmann and G.Bergmann, Phys.Rev.Lett.83, 2417 (1999)

[18] G.Bergmann and M.Hossain, Phys.Rev.B60, 15621 (1999)

[19] G.Bergmann and M.Hossain, Phys.Rev.Lett. 86, 2138 (2001)

[20] Mohamed Hossain and Gerd Bergmann, Eur.Phys.J B26, 7 (2002)

[21] Funing Song and Gerd Bergmann, Phys.Rev.Lett. 88, 167202-1 (2002)

[22] Douglas Garrett and Gerd Bergmann, Phys.Rev.B66, 224407 (2002)

[23] Funing Song and Gerd Bergmann, Phys.Rev. B68, 094403 (2003)

[24] P.G.de Gennes, Rev.Mod.Phys. 36, 1, 225 (1964)

[25] N.W.Ashcroft and N.D.Mermin, Solid State Physics, Saunders College, Philadelphia 
[26] M.Ashida, J.Hara and K.Nagai, Phys.Rev. B45, 828 (1992)

[27] L.P.Gorkov, Pis'ma Zh.Eksp.i.Teor. Fiz. 37, 1407 (1959), JETP 10, 998, (1960)

[28] G.Lueders and K.-D.Usadel, in Springer Tracts in Modern Physics, Vol. 56, 1 (1971), ed. G.Hoehler, Springer Verlag Berlin, Heidelberg, New York 1971

[29] G.Bergmann, Z.Physik 234, 70 (1970) 


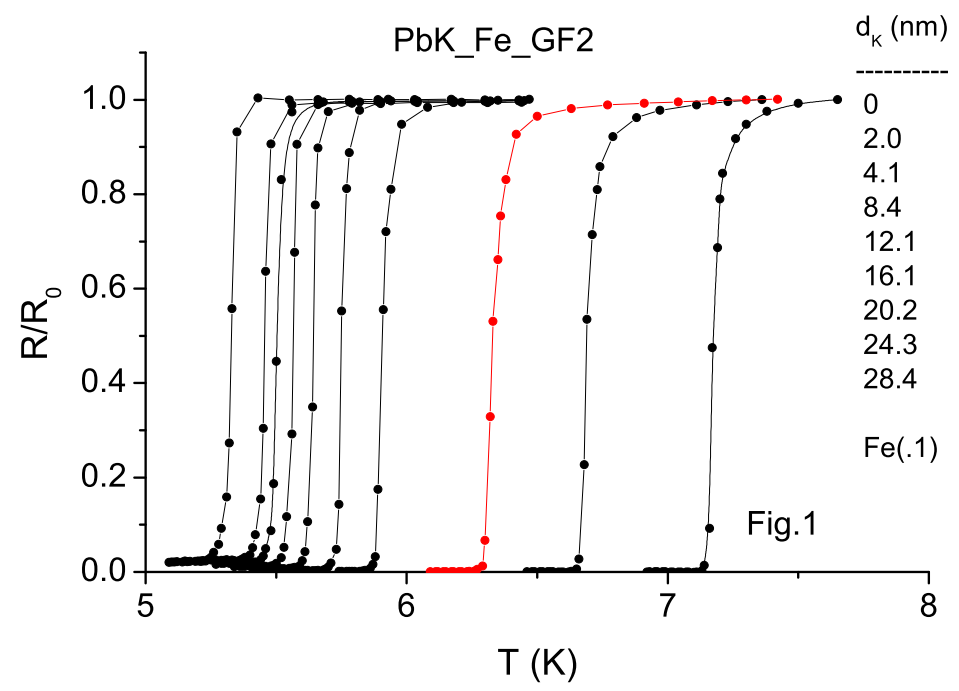




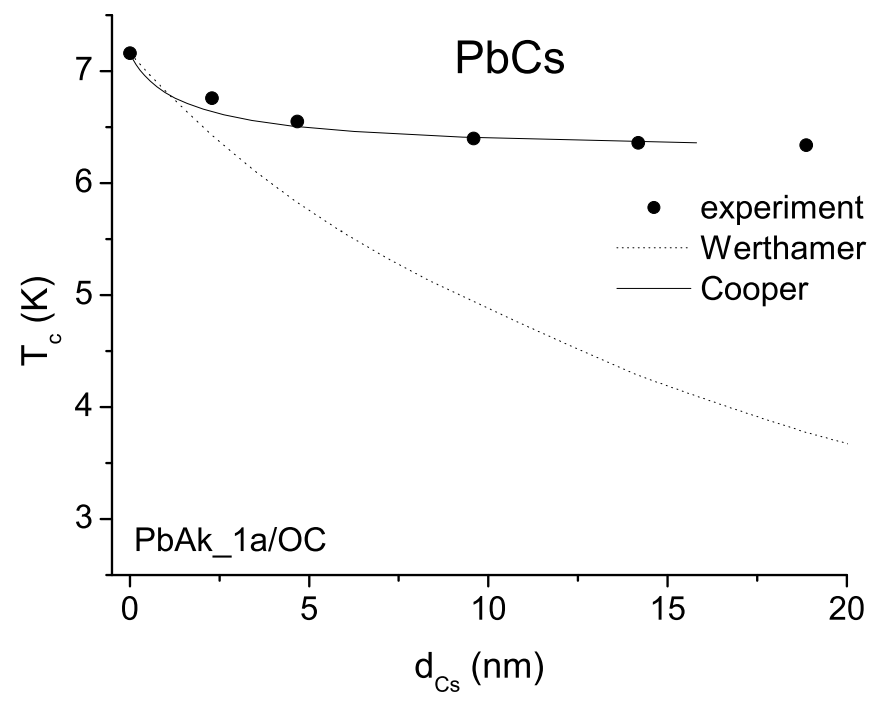




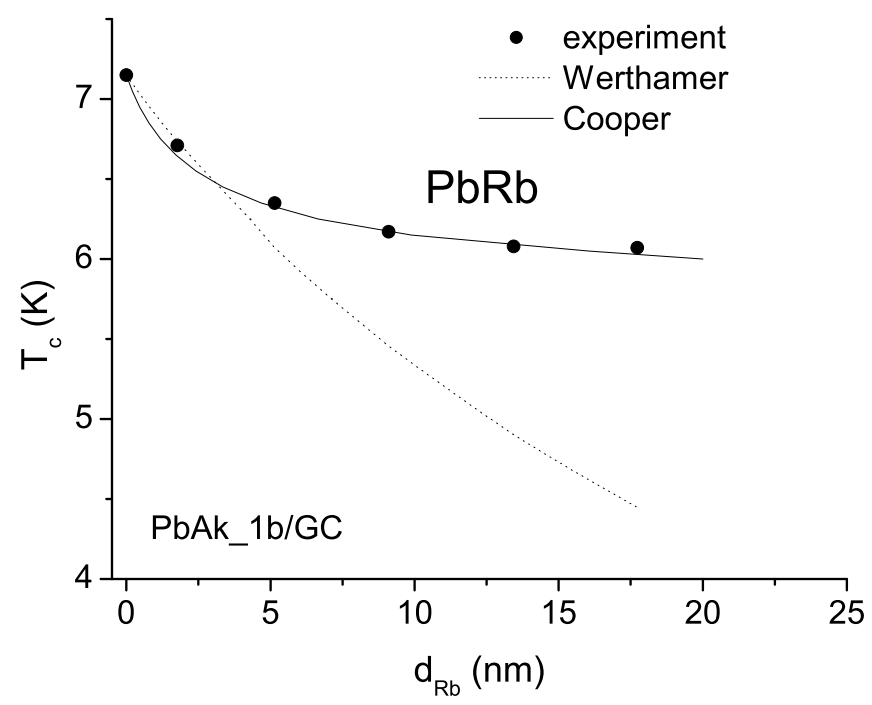




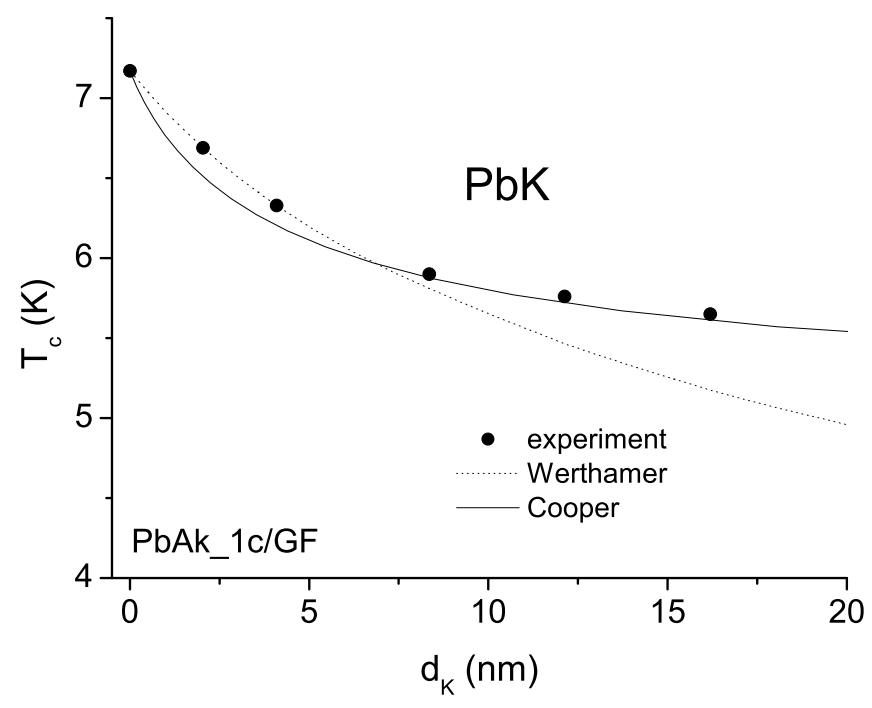



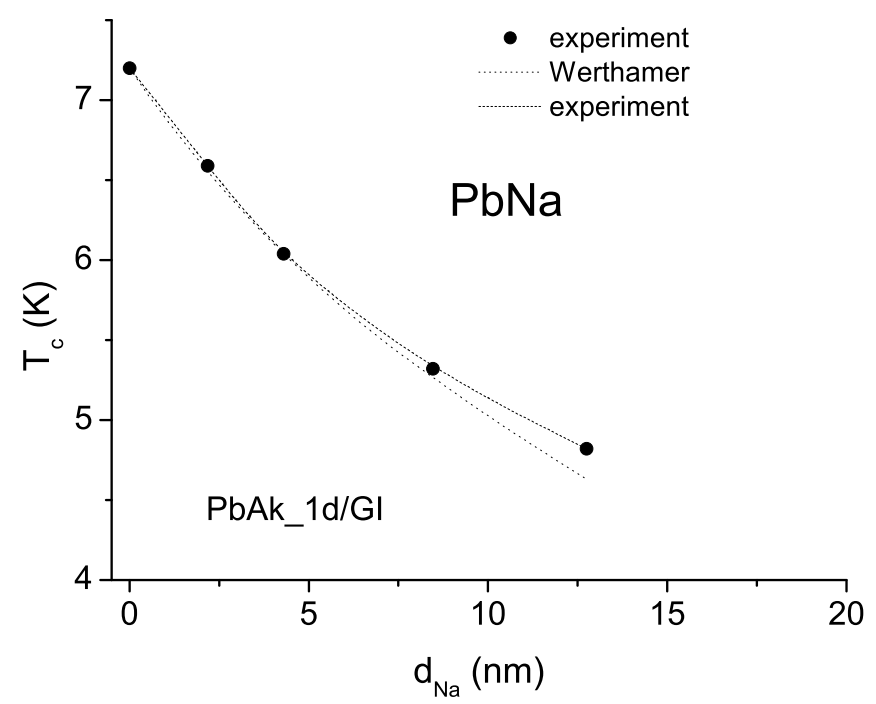


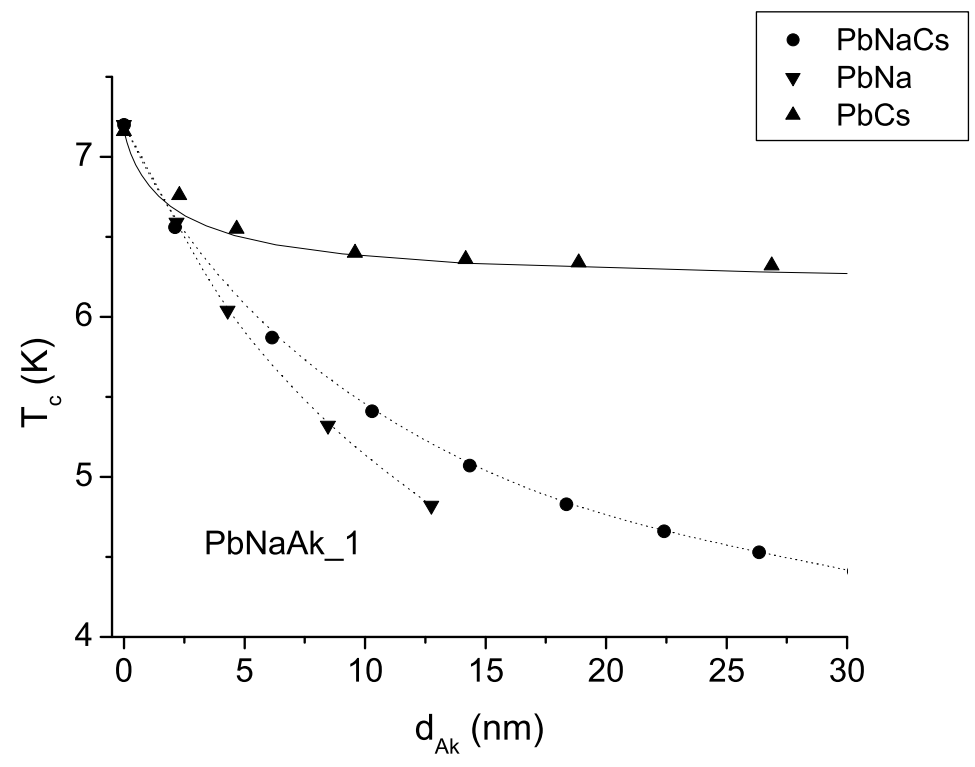

